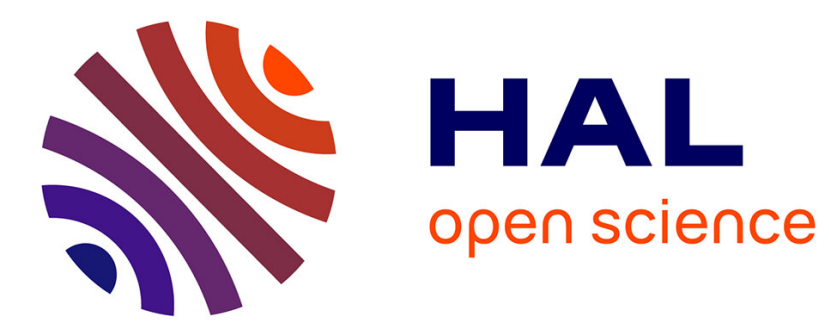

\title{
Hypersonic flows for reentry problems
}

Remi Abgrall, Jean-Antoine Desideri, Michel Mallet, Jacques Periaux, Pierre Perrier, Bruno Stoufflet

\section{To cite this version:}

Remi Abgrall, Jean-Antoine Desideri, Michel Mallet, Jacques Periaux, Pierre Perrier, et al.. Hypersonic flows for reentry problems. [Research Report] RR-1721, INRIA. 1992. inria-00076959

\section{HAL Id: inria-00076959 https://hal.inria.fr/inria-00076959}

Submitted on 29 May 2006

HAL is a multi-disciplinary open access archive for the deposit and dissemination of scientific research documents, whether they are published or not. The documents may come from teaching and research institutions in France or abroad, or from public or private research centers.
L'archive ouverte pluridisciplinaire HAL, est destinée au dépôt et à la diffusion de documents scientifiques de niveau recherche, publiés ou non, émanant des établissements d'enseignement et de recherche français ou étrangers, des laboratoires publics ou privés. 
UNITÉ DE RECHERCHE INRIA-SOPHIA ANTIPOLIS

Institut National de Recherche en Informatique et en Automatique

2004 route des Lucioles B.P. 93

06902 Sophia-Antipolis France

\section{Programme 6}

Calcul scientifique, Modélisation et Logiciel numérique

SYNTHESIS OF THE WORKSHOP ON HYPERSONIC FLOWS FOR REENTRY PROBLEMS, PARTS I \& II

Rémi ABGRALL Jean-Antoine DESIDERI Michel MALLET Jacques PÉRIAUX Pierre PERRIER Bruno STOUFFLET

Juin 1992 


\section{Synthesis of the Workshop on HYPERSONIC FLOWS FOR REENTRY PROBLEMS}

held in Antibes, France, in January 1990 and April 1991

Rémi Abgrall, Jean-Antoine Désidéri, INRIA Sophia Antipolis, B.P. 109, 2004 Route des Lucioles - 06561 Valbonne Cedex (FRANCE),

Michel Mallet, Jacques Périaux, Pierre Perrier and Bruno Stoufflet, Dassault Aviation, 78 Quai Marcel Dassault - 92214 Saint-Cloud (FRANCE).

\section{Motivations}

One of the most challenging problems of modern aerospace engineering is undoubtedly the prediction of hypersonic flows around reentry vehicles. Indeed this challenge has many aspects : defining the appropriate physical model for these flows is the first difficulty since very complex physical and chemical phenomena take place during the reentry phase; moreover, temperature, velocity and enthalpy are very high, density very low, making the reentry conditions very difficult to reproduce in ground-based experiments.

Thus, in the past two decades, important efforts in Computational Fluid Dynamics have been made employing supercomputers to simulate these complex flows. However, most simulation tools were originally designed for transonic or supersonic flow applications and later extended; these usually contain imperfect models, and also still have to be improved to permit the accurate prediction of hypersonic flows.

This situation has motivated strong international cooperative efforts including three Europe / US Short Courses on Hypersonics [1] [2] and two sessions (Parts I and II) of the scientific workshop organized by INRIA and GAMNI [3] which took place in Antibes, France.

Part I brought together a group of experts active in Hypersonics, and promoted direct comparisons of codes and experiments. Eight critical test problems involving well-defined 2-D and 3-D steady flows governed by specified P.D.E.'s and boundary conditions were submitted to computation. CFD results have been compared altogether, and confronted to experiments when available. This led to a first estimation of CFD capabilities in Hypersonics as reported in the workshop proceedings [3].

The motivation for a second session, Part II, of the same workshop was to propose a more thorough study of each test problem by reducing the test-cases to a representative subset, by improving the definition of some of them (e.g. when possible the description of the nonuniformity of the inflow in the experiment was provided to the CFD community), and also by adding a few novel test-cases associated with new experiments. In addition, computational contributors were urged to perform calculations on refined meshes, and to study grid refinement effects, and to control artificial diffusion.

In summary, the main objective of Part II was to improve the quality of both experimental and computational results, and evaluate numerical codes by code-to-code or code-to-experiment comparisons, and permit a better confidence in the prediction tools to be used in the design of hypersonic vehicles.

The presentation is aimed at reporting the main conclusions as they were expressed at the meeting by experts, including some of their recommendations regarding the validation of numerical codes. 


\section{Development of the meetings}

Both sessions (Parts I and II) included ten invited lectures related to specialized issues in experimentation, modeling and computing in Hypersonics.

The rest of the workshop was developed around specific test problems. During reentry, three main types of flow are encountered :

- rarefied gas flow,

- reacting flow,

- non-reacting flow .

The governing equations to be solved are then respectively

- the Boltzmann equations,

- the Euler or Navier-Stokes equations (or other intermediate models such as the thin layer or parabolized, viscous shock layer or coupled Euler/Boundary layer models) augmented of a chemistry model,

- the Navier-Stokes equations possibly combined with a turbulence model.

The following eight critical problems were proposed for experimentation and computation :

Problem 1: Flow over a slender cone

Problem 2: Base flow

Problem 3: Flow over a 2-D ramp

Problem 4: Flow over a 3-D obstacle

Problem 5: Corner flow

Problem 6: Flow over a Double-Ellipse/Ellipsoid

Problem 7: Flow over a delta wing

Problem 8: Non-equilibrium flow in an arc-jet or a shock tube.

The themes of theses problems are turbulence transition ( 1 and 2 ), heat-transfer prediction (3, 4 and 5), real-gas effects (6), vortex or rarefaction effects (7), thermal non-equilibrium (8).

For each problem, computational contributions were presented following the presentation of experimental results, and preliminary critical analyses were made by experts during the program, which also included poster sessions.

In tables 1 and 2 below, are listed the number of experimental or computational contributions to the various test problems. The numbering of these problems was modified (to roman figures) for Part II to facilitate the suppression of certain test cases associated to given problems, and the introduction of novel ones. The details of the definition of the test cases can be found in the announcement brochures (Part I and II), and in the proceedings of the workshop [3].

Table 1: Participation in Part I

\begin{tabular}{|l|r|r|r|r|r|r|r|}
\hline & & Pbs 1-2 & $3-4$ & 5 & 6 & 7 & 8 \\
\hline Experiments & 12 & 1 & 3 & 1 & 3 & 3 & 1 \\
\hline Computations: & & & & & & & \\
Europe & 56 & 2 & 12 & 1 & 29 & 8 & 4 \\
USA & 22 & 3 & 4 & 2 & 7 & 4 & 2 \\
Japan & 3 & 1 & - & - & 2 & - & - \\
Australia & 2 & - & 1 & - & 1 & - & - \\
\hline TOTAL & 95 & 7 & 20 & 4 & 42 & 15 & 7 \\
\hline
\end{tabular}


Table 2: Participation in Part II

\begin{tabular}{|l|r|r|r|r|r|r|r|}
\hline & & Pbs I-II & III-IV & V & VI & VII & VIII \\
\hline Experiments & 8 & 1 & 1 & 1 & 1 & 3 & 1 \\
\hline Computations: & & & & & & & \\
Europe & 65 & 2 & 20 & 2 & 19 & 11 & 11 \\
USA & 8 & - & 3 & - & 1 & 4 & - \\
Australia & 2 & - & 1 & - & - & 1 & - \\
\hline TOTAL & 83 & 3 & 25 & 3 & 21 & 19 & 12 \\
\hline
\end{tabular}

It appears that if a substancial effort was made (mostly by the European Community) on the double-ellipse/ellipsoid problem for Part I, and to a lesser extent on the ramp problem, a noticeable shift of interest was observed for Part II on the delta wing and the high-enthalpy flow problems.

\section{Invited talks}

During each session, the technical presentation of test problem results were completed by invited lectures given by leading scientists on topics related to modeling experimental aspects and numerical simulations of hypersonic flows. The list of these talks is given below :

\section{$\underline{\text { Part I : }}$}

- Inaugural Talk : Introduction to the Workshop, J. Carpentier (ONERA, France),

- Comments on experiments for computational validation for fluid dynamic predictions, S. M. Bogdonoff (Princeton, USA),

- Hypersonic Stability and Transition, E. Reshotko (Case Western Reserve University, USA),

- Scattering kernels for gas-surface interaction, C. Cercignani (Politecnico di Milano, Italy)

- CFD for hypersonic air-breathing aircraft, J. L. Thomas, D. L. Dwoyer, A. Kumar (NASA Langley, USA),

- Similitude in hypersonic aerodynamics, H. Viviand (ONERA, France),

- Gas-kinetical and Navier-Stokes simulations of reentry flows, H. Oertel Jr. (University of Braunschweig, Germany),

- Numerical simulation of Boltzmann flows of real gases - Accuracy of models used in the Monte-Carlo methods, K. Nanbu (NAL, Japan),

- Intrusive and non-intrusive measurements of flow properties in arc jets, C. D. Scott (NASA JSC Houston , USA),

- CFD for hypersonic propulsion, L. A. Povinelli (NASA Lewis, USA),

- Experimental simulation of high-enthalpy real-gas effects, H. Hornung (Cal'Tech, USA).

$\underline{\text { Part II : }}$

- Inaugural talk : Fundamental and applied research in space applications, R. Pellat (CNRS and CNES, France),

- Experimental studies in shock-shock and shock-boundary-layer in laminar and turbulent hypersonic flows, M. S. Holden (Calspan, USA),

- Some viscous interactions affecting the design of hypersonic intakes and nozzles, J. L. Stollery (Cranfield Institute of Technology, UK),

- Reacting viscous flow and gas-surface interaction modeling, J. Warnatz (University of Stuttgart), - Shock-tunnel experiments involving real-gas effects at Australian Universities, M. Stalker (University of Queensland, Australia), 
- Numerical simulation of scramjet engine flowfield, A. Kumar (NASA Langley, USA),

- The role of applied mathematics in the numerical simulation of hypersonic flows, O. Pironneau (University of Paris VI, France),

- The German hypersonic technology program Sänger, H. A. Hertrich (Ministerialrat BMFT, Germany),

- Hypersonic reasearch at German universities, E. Krause (RWTH Aachen, Germany),

- CFD code validation experience, J. G. Marvin (NASA Ames, USA).

\section{Analysis of Results}

\subsection{Synthesis of results for Problems I and II}

\subsubsection{Experiments}

(Figure 1)

P. A. Denman, J. K. Harvey and R. Hillier (Imperial College, UK) provided stanton number, Mach number and pitot pressure along the cone and the base. They measured pitot pressure and velocity profiles at two different stations on the cone. The experiments were conducted in the Imperial College No. 2 gun tunnel.

Measurements exhibited a small streamwise gradient, a simple model of which was provided for computations for Part II.

The challenge in the first experimental test case was to reproduce a very simple conical flow.

\subsubsection{Computations}

One of the major problems for Part I relied in the location of transition. This transition was arbitrarily fixed for Part I, and specified by the experimentalists for Part II. Important discrepancy between computations and experiments despite sensitivity to grid, wall temperature, turbulence model had been studied. The Stanton number has been slightly over-estimated (of some $10 \%$ ) by certain computations.

The accurate calculation of the Stanton number in Problem II is critically dependent on the location of the reattachment point. Some $20 \%$ discrepancy with the experiment in this parameter was observed in certain computations. The computed separation region was found too small and making a questionable angle with the wall at the edge of the base.

\subsection{Synthesis of results for Problem III (flow over a $2 \mathrm{D}$ ramp)}

\subsubsection{Experiments}

J. Delery and M. C. Coët conducted the tests in the R2Ch and R3Ch blow-down wind-tunnel of the ONERA Chalais-Meudon establishment. They provided Schlieren photographs, wall heat transfer and wall pressure distributions. The wedge-flow experiments were made at Mach number 5 and 10 to study the interaction pattern at both low and high Reynolds numbers.

G. Simeonides and J. F. Wendt (Von Karman Institute, Belgium) conducted experiments in the long-shot heavy-gun piston gun-tunnel at the VKI with a flat-plate $/ 2-\mathrm{D} 15^{\circ}$ ramp model and provided pressure and heat transfer distribution along the centerline yielding an indication of the transition in the reattachment region. 


\subsubsection{Computations}

(Figure 2)

Navier-Stokes computations of a hypersonic flow over a 2-D ramp are compared to one another and to experiments. The importance of grid density was clearly put into evidence as concerns the prediction of separation lengths. Difficulties in achieving well-defined experimental conditions, particularly fully laminar conditions at reattachment were discussed. Considerable variations in the predicted location of the reattachment region were observed, and one alleged explanation is that the experiment is characterized by a transitional reattachment. Some contributors showed that putting a switch to turn on the turbulence model resulted in a much better agreement with the experiment in the ramp region.

Four test cases were proposed:

- test cases III- 1 and III- 2 are characterized by a low value of the Reynolds number (Rey= 143800). This was chosen to make the test case less sensitive and to reduce the likelihood of transition at reattachment.

- test case III.3 was already proposed for Part I of the workshop (problem 3.5), a large amount of information is already available but improved results were expected.

- test case III.4 is chosen from a set of tests performed by Holden and has already been used extensively for validation.

24 numerical contributions were presented at the meeting, this is enough to get a good idea of how CFD tools can perform on this problem. Various numerical approaches were used including centered + artificial viscosity, upwind and Galerkin least squares methods.

Very good agreement is observed for problem III.1 which was easy because of the absence of recirculation.

Reasonably good agreement is observed for problem III.2 which was designed to be easier for numerical contributors (e.g. on skin-friction coefficient). It should be noted that the structure of the flow is quite different than what is observed for a standard (less viscous) case: the pressure skin friction and heat flux do not reach a maximum on the deflected plate. Results can be gathered into two groups that differ on the size of the recirculation.

Experimental results are not available at present to discriminate between both sets. Even though the overall agreement is good, it is somewhat disappointing to observe that for such a viscous case large differences still exist.

For problem III.3, a very large discrepancy is still observed. The most striking results of the computations is the prediction of an excessively large region of recirculation. A number of explanations can be considered:

- the thin layer Navier Stokes simplification may not be valid,

- the two dimensional character of the flow was questioned (however, the deflection angle is low and no experimental evidence of 3D flow was observed (influence of side plates),

- the proper choice of the grid is critical. This was not addressed in enough detail and it was not clear that the solutions presented were grid independent (in the sense that nodes should be added until addition of nodes does not affect the solution; better agreement would be observed if calculations were performed again on refined grids),

- transition near the reattachment point is a possibility,

- the presence of Görtler vortices on the ramp is still an open question. 


\subsubsection{Conclusions}

The problem of the calculation of the flow over a ramp is more difficult than could be anticipated. Nevertheless, at the second meeting, we observed in certain contributions gridconverged solutions, convergence acceleration by Multigrid, and a more careful attention given to the grid density in the boundary layer. Provided that sufficient attention is given to the choice of a proper mesh, we still feel that accurate solutions can be obtained.

\subsection{Synthesis of results for Problem IV (flow over a 3D ramp)}

\subsubsection{Experiments}

An experiment was made at Mach number 10 by Delery et al. for a $30^{\circ}$-swept 3 - $\mathrm{D}$ obstacle. They provided surface flow visualizations, wall heat transfer and pressure distributions illustrating very extended zone of separated flow.

Again, the authors indicate that reattachment may have been transitional rather than laminar.

\subsubsection{Computations}

(Figure 3)

Two results were available at the meeting.

The contribution by Jouet et al. uses a finite volume approach with Roe's fluxes and Van Leer's MUSCL approach. The grid includes 300000 nodes. Results are not fully converged and the initialization leads to an overprediction of the size of the recirculation.

Hassan et al use a Taylor Galerkin FEM and a mesh of 80000 nodes. The calculation slightly underpredicts the value of the Stanton number.

In addition, a contribution by Venkatapathy in which the full compressible Navier-Stokes equations were solved for a problem close to the workshop specification, indicated good qualitative features (separation line position, separation line curvature).

\subsection{Synthesis of results for Problem V (corner flow)}

\subsubsection{Experiments}

(Figure 4)

A. Henckels and F. Maurer (DLR Köln, Germany) measured the heat flux for the corner flow case at the H2K hypersonic wind tunnel with an infrared thermovision camera system in connection with image processing.

We note that the experimental data were very accurate and supported by a large amount of local measurements.

\subsubsection{Computations}

Two contributions only were available at the meeting.

Lacor et al. use a finite volume approach with central difference and artificial diffusion. The mesh consists of 34000 nodes.

Panaras et al. use an upwind finite difference scheme based on a TVD method. A good visualization of the leading edge shocks interaction is provided.

The agreement between the contributions is poor. 


\subsection{Synthesis of results for Problem VI.1 (Inert-gas flow over Dou- ble Ellipse/Ellipsoid)}

\subsubsection{Experiments}

D. Aymer, T. Alziary (CEAT Poitiers, France) and L. De Luca, G. Carlomagno (Università di Napoli) provided Schlieren and wall streamline visualizations, wall pressure and heat flux measurements at the H210 wind-tunnel facility. The heat flux was measured by infrared thermocouple using the thick wall technique.

\subsubsection{Computations}

(Figure 5)

The 3-D double-ellipsoid shape has shown to be a very good test of complex flow in hypersonics (small nose radius, bow shock, secondary shock at the canopy, complex pattern of separated flow with angle of attack). A lack of detailed flowfield measurements did not permit a precise check of the topology of the flow away from the wall.

At the first meeting, all solutions exhibited a good performance with respect to the prediction of pressure concerning both the comparison between each other and with experimental data. As expected the inviscid flow simulations do predict well windward pressure distributions in hypersonic flows.

Concerning the prediction of the embedded shock, the shock seemed generally predicted detached.

Appreciable scatter was observed on boundary layer quantities such as skin-friction coefficient and Stanton number, probably due to differences in grid resolution and numerical diffusion terms. It is surprising to observe scatter not only in flows with embedded shocks but also in those along windward surfaces.

During Part II, the overall quality of the Navier-Stokes contributions improved due to efforts made in constructing better meshes : number of nodes in the boundary layer, first node close to the wall, better control of cell aspect ratio.

\subsection{Synthesis of results for Problem VI.2/3 (Reacting-gas flow over Double Ellipse/Ellipsoid)}

\subsubsection{Experiments}

(Figure 7)

M. Vetter, H. Olivier and H. Grönig (RWTH Aachen, Germany) measured Cp and Stanton number wall distributions along an 18-cm long double-ellipsoid subject to real-gas effects and catalytic wall.

They also provided Schlieren pictures of the reacting flow around a 50-mm radius sphere for $T_{0}=1500 \mathrm{~K}$ and $4600 \mathrm{~K}$.

\subsubsection{Computations}

(Figures 6 and 8)

Synthesizing the results of Part I, we concluded that several computations of reactive flows over the double ellipse (ellipsoid) had been proposed. They mostly dealt with inviscid flow in chemical equilibrium or non-equilibrium. For equilibrium flows, the agreement in shock locations between the various contributions was comparable with that of inert-gas computations with comparable meshes; the discrepancies between the various contributions were very small in 
temperature but somewhat larger in Mach number and composition. For non-equilibrium flow, some discrepancy in the main shock location had been observed in two shock fitting procedures. Conventional codes were observed to predict wall values of temperature and composition vastly different from equilibrium state because, in the theoretical inviscid solution, the gradient of temperature or composition normal to the wall is infinite ("chemical layer"). Two procedures had been shown to remedy this problem: one by mesh refinement, the other by post-processing of the conventional solution. Both essentially agreed.

Part II confirmed these facts. Nevertheless the agreement between the various computations of 2-D non-equilibrium inviscid flows were somewhat better in Part II due to the authors efforts in mesh-refinement studies. Concerning, 2-D viscous non-equilibrium flows, although flowfields are comparable, discrepancies with the experimental data are substancial in wall distributions; this is true even for the wall pressure distribution, traditionally less controversial. Mesh refinement near the wall was evidently found very critical, but naturally more importantly the treatment of the boundary condition (catalyticity). The latest results by Salvetti et al. are much closer to the experiment. A general observation concerning Navier-Stokes calculations, was that the scatter in the stagnation point heating possibly due different models was of the order of $25 \%$. A boundary-layer calculation for the double-ellipse case was also presented and appear to be a reasonable less costly alternative when applicable.

Concerning equilibrium-air flows, at least three Navier-Stokes solutions were presented and five dealing with inviscid flow. In addition, at the meeting was shown a 3-D boundarylayer calculation of non-equilibrium flow.

\subsection{Synthesis of results for Problem VII (Delta Wing)}

\subsubsection{Experiments}

M. Linde (FFA, Sweden) investigated the leeside flow over a sharp delta wing in terms of vortical features and shock induced separation test in the FFA HYP 500 hypersonic wind tunnel with surface oil flow and pressure measurements. The flow is conical at the forward part of the wing whereas on the rear part there is a separated region with recirculating flow.

J. Allègre, X. Hériard Dubreuilh and M. Raffin (CNRS/SESSIA, Laboratoire d'Aérothermique de Meudon) provided the density flowfield data and aerodynamic forces for the rarefied-flow case around the delta wing by the electron-beam technique. The experimental conditions were characterized by two rarefaction levels $\left(M_{\infty}=20.2, R e_{L}=2050\right.$ and $\left.M_{\infty}=20, R e_{L}=8380\right)$.

Experiments were carried out in the hypersonic vacuum wind tunnel V2G in Göttingen by Ch. H. Chun (DLR Göttingen, Germany) around a different delta wing with the freestream conditions : $M_{\infty}=24, R e_{L}=2.9 \times 10^{4}$, at angles of attack $\alpha$ between $0^{\circ}$ and $30^{\circ}$ for the heat flux measurements, and $\alpha$ between $0^{\circ}$ and $40^{\circ}$ for the force measurements,

H. Legge (DLR Göttingen, Germany) provide force and heat transfer measurements of rarefied flow over a mini delta wing at large Knudsen number showing complete accommodation. The covered rarefaction range is especially useful for comparisons with Monte Carlo calculations. The rarefied flow on the delta wing was of high quality. For this reason, a duplication of the test did not seem necessary.

\subsubsection{Computations}

(Figures 9-10)

The understanding of vortex structures and leeside separations constitutes one of the major element in the analysis of hypersonic flows around reentry vehicles in atmosphere. As test case supporting this objective, the computation of the flow around a Delta Wing was proposed with two models (inviscid and viscous simulations). 
For Part I, the geometry of a Delta Wing with a sharp nose was defined and a test case (VII.1 for inviscid flow and VII.2 for viscous one) based on experimental conditions was considered $\left(M_{\infty}=7.15\right.$ and $\left.\alpha=30^{\circ}\right)$. In the second part, a blunt nosed Wing has been defined corresponding to a model tested at DLR at $M_{\infty}=8.7$ and $\alpha=30^{\circ}$ (test cases VII.3 and VII.4). A more thorough analysis of such problems was then possible with the two sets of solutions.

For the first geometry, we were concerned with a wedged windward side and a general shape close to a conical-like shape whereas the second one consisted in a curved windward side with a general shape far from being a conical-like one. Consequently, the flow solution is more or less autosimilar in $x$-planes for the first geometry whereas solution evolves with the space direction $x$ for the second one. The sharp nose is good leeside vortex generator while no vortex sheet appears for the blunted nose wing. These remarks are illustrated by numerical results provided by ONERA.

For Part II, contributors concentrated much on the test cases VII.3 and VII.4. Eight computations wer performed for the inviscid case with meshes ranging from 110,000 to $2,000,000$ nodes (this contribution can be considered as a reference solution) for structured grid approaches and of about 70,000 nodes for the two unstructured grid methods. Concerning test case VII.4 (viscous flow), seven contributors participated with meshes ranging from about 300,000 to $1,000,000$ nodes.

\subsubsection{Conclusions for the inviscid flow solutions}

Although a good agreement was found in the evaluation of pressure coefficients, two families of solutions were obtained when considering Mach number distribution on the leeside. Some contributions show a large Mach number peak due to the very strong expansion at the leading edge. This peak was found in the ONERA computation with the very fine grid but no real conclusive interpretation has yet been given. A strong grid sensitivity on leeward was revealed for all approaches and in particular in the DASSAULT solutions. It has been noticed that a total temperature loss of about $3.5 \%$ was obtained among the most accurate solutions.

\subsubsection{Conclusions for the viscous flow solutions}

Comparisons of the numerical solutions with the experimental curves were available. Computed Stanton numbers have been found noticeably higher than the experimental values. A good qualitative agreement was however found.

This test case was computed with different flow modelizations (Euler solver with boundary layer analysis, Parabolized Navier-Stokes solver and Navier-Stokes solvers) and all the numerical results provide a remarkable numerical agreement for Stanton numbers.

\subsubsection{Conclusions for the rarefied flow solutions}

(Figure 11)

The classical Bird DSMC method and a variant, which is referred to as the Finite-Pointset Method (FPM) introduced by the University of Kaiserslautern, were employed to compute rarefied gas flow cases. It is evident that both of the computations are of very high standard and they yield almost identical results for the overall flux quantities. It has been demonstrated that these methods are capable of predicting complex flows in which molecular non-equilibrium effects are present. 


\subsection{Synthesis of results for Problem VIII (Arc Jet and Shock Tube)}

\subsubsection{Experiments}

The purpose of this test case was to obtain a better understanding of the non-equilibrium flowfield of a high-enthalpy plasma tunnel which serves to study the thermal protection system of future spacecraft.

J. M. Léger, J. F. Coudert, A. Grimaud and P. Fauchais (University of Limoges, France) measured the temperature fields of d.c. nitrogen plasma jets in a controlled atmosphere chamber filled with nitrogen at pressure between 13 and $100 \mathrm{KPa}$ by emission spectroscopy. Nonequilibrium effects were shown with significant differences between rotational, population and excitation temperatures. Recently, they provided the entrance velocity distribution near the axis for a better specification of the initial condition.

\subsubsection{Computations}

(Figure 12)

\section{Arc Jet}

The comparison between computational contributions could not be performed in sufficient details because of the differences in the assumed chemistry composition at the inlet cross section. Comparing the calculated values with the measurements, the trends were the following :

- the calculated values of $T_{\text {rot }}$ and $T_{\text {ele }}$ seem underestimated along the jet axis, - the difference of this two variables was less in the computations than in the experiments.

The computational validation has recently been improved since additional velocity distribution in the plasma was made available from the experiments. CFD tools seem more powerful than anticipated for a so complex and difficult test case. A logical follow up of this test case would be an arc jet with air surrounded by expanding nozzle to better simulate industrial plasma tunnels.

Nozzle Flow

The purpose of this test case was to validate the computational tools which are mandatory for the correct interpretation of the measurements made on models in high enthalpy facilities.

As no experimental results are available, only code-to-code comparisons have been made. From the computations, it appeared that the results are mostly sensitive to :

- numerical approximation (scheme and mesh),

- inclusion of thermal non-equilibrium;

however, the following parameters seem to play a lesser role :

- choice of vibrational model, - inclusion of $\mathrm{V}-\mathrm{T}, \mathrm{V}-\mathrm{V}$ exchange models,

- 2-D approximation rather than 1-D.

Finally, it was noted that a systematic sensitivity study of the chemical model would be necessary.

\section{Conclusion and Further Comments}

The Antibes Workshop has permitted a better assessment of some of the performance and limits of modern CFD tools applied to hypersonic flow computations. The necessity of bringing together fluid mechanicists, specialists of physico-chemical modeling, numerical analysts, applied mathematicians is obvious since the full understanding of hypersonic flows cannot be achieved by either community alone. This type of scientific validation workshop contributes to reduce 
uncertainties in the design of spaceplanes [5]. Evidently, a limited number of experiments, or alternatively, an approach based solely on computations, would not permit a total confidence in hypersonic design tools.

By the number of participants, the quality of experimental and computational data obtained despite the difficulty involved, and the material presented, this first open workshop in Hypersonics has been a good achievement.

From a technical point of view, it appeared that the computation of Eulerian flows was now well achieved by current approximation methods provided sufficient efforts were devoted to the mesh quality in particular. However, the computation of complex viscous and/or rarefied flows, possibly turbulent or reactive, or both, is still a great challenge. These require good numerics, but also adequate models still under investigation, and experiments of high standard for the validation.

The validation process will continue using some of the data collected at the occasion of this workshop. A European Hypersonic Data Base (EHDB) is currently being set up at INRIA Sophia Antipolis, France and will be accessible by networks. The objective is to offer experts in this field a means to evaluate novel CFD methodologies using existing data related to the workshop test problems.

The great challenge for the forthcoming years with available massively parallel computing facilities is to conduct experiments and computations of three-dimensional complex flows including transition, separation and real-gas effects [4]. This will undoubtedly be the focus of the future workshops.

\section{Acknowledgements}

The authors are grateful to invited speakers, experimentalists and computational contributors who made this workshop a success by their continuous and friendly assistance. We are particularly indebted to the experts who prepared the syntheses of Part I, and the Chairmen of Part II; their role was essential in the preparation of this article.

\section{References}

[1] J. J. Bertin, R. Glowinski, J. Périaux, Eds., Proc. of the First Joint Europe / US Short Course in Hypersonics, Paris, France, December 1987, Vol. I and II, Progress in Scientific Computing, Birkhäuser, Boston, 1989.

[2] J. Ballman, J. J. Bertin, J. Périaux, Eds., Proc. of the Second and Third Joint Europe / US Short Course in Hypersonics, Colorado Springs U.S.A., January 1989, and Aachen Germany, October 1990, Progress in Scientific Computing, Birkhäuser, Boston, to appear.

[3] J. A. Désidéri, R. Glowinski, J. Périaux, Eds., Hypersonic Flows for Reentry Problems, Vol. I and II, Springer-Verlag, 1991, Vol. III in preparation.

[4] T. L. Holst, M. D. Salas, R. W. Claus, The NASA Computational Aerosciences ProgramToward Teraflops Computing, AIAA 92-0558, Reno 1992.

[5] P. Perrier, Aerodynamic and Aerothermal Challenges for the design of the HERMES Spaceplane, 70th Fluid Dynamics AGARD Panel Meeting and Symposium on Theoretical and Experimental Methods in Hypersonic Flows, Torino, Italy, May 4-8, 1992. 
Figure 1 : Cone and Base Stanton number

Figure 2 : Stanton number (results from Part I, case 3.5, and Part II, case III.3).

Figure 3 : Stanton number on sections $\mathrm{A}$ and $\mathrm{C}$ (test case IV.1)

Figure 4 : Stanton number in the sectional cut $0.2 \mathrm{~m}$ behind leading edge; oil flow visualization (Henckels et al.).

Figure 5: Wall Stanton number and skin friction coefficient in symmetry plane - Navier Stokes inert flow (from Part I).

Figure 6 : 2-D non-equilibrium Eulerian flow computations (temperature and atomic nitrogen on body surface) (test case 6.2 .3 )

Figure 7 : Wall pressure coefficient and Stanton number in the symmetry plane (Vetter et al.).

Figure $8: 2-D$ non-equilibrium Eulerian flow computations (Mach number and temperature on body surface) (test case VI.2)

Figure 9 : Mach, $\mathrm{Cp}$, cross velocity (refined grid results) contours at $\mathrm{x} / \mathrm{c}=0.5$ and 0.8 of Eulerian flow around the sharp delta wing (test case VII.1) (Le Toullec et al.).

Figure 10: Comparison of Stanton number distributions in spanwise direction, $110 \mathrm{~mm}$ and 190 mm behind tip (test case VII.4) (Chaput et al., Menne, Riedelbauch; Chaput et al., Srinivasan et al., Schröder et al.).

Figure 11 : Pitching moment coefficient and density flowfield in the plane of symmetry at $\alpha=30^{\circ}$; calculated results compared with experimental data (test case VII.5).

Figure 12 : Electronic concentration $(\mathrm{x}=3 \mathrm{~mm})$; translational temperature $(\mathrm{x}=.013 \mathrm{~m})$ (VIII.1); Temperatures along symmetry axis (Mulard et al.); 2-D viscous results (VIII.2). 